Proceedings of the 1999 IEEE

International Conference on Robotics \& Automation

Detroit, Michigan • May 1999

\title{
ProVAR Assistive Robot System Architecture
}

\author{
H.F.M. Van der Loos ${ }^{1}$, J.J. Wagner ${ }^{2}$, N. Smaby ${ }^{2}$, K. Chang ${ }^{3}$, O. Madrigal ${ }^{2}$, L.J. Leifer ${ }^{2}$, O. Khatib ${ }^{3}$ \\ ${ }^{1}$ Rehabilitation R\&D Center, VA Palo Alto Health Care System; \\ ${ }^{2}$ Dept. Mechanical Engineering, ${ }^{3}$ Dept. Computer Science, Stanford University
}

\begin{abstract}
This paper describes the implementation of a robot control architecture designed to combine a manipulation task design environment with a motion controller that uses the Operational Space formulation to define and implement arm trajectories and object manipulation. The ProVAR desktop manipulation system is an assistive robot for individuals with a severe physical disability such as quadriplegia as a result of a high-level spinal cord injury. ProVAR allows non-technical operators access to the robot's capabilities through a directmanipulation simulation/preview user interface. The novel interface concept is based on two built-in characters (Jiminey and Pinocchio) to play the roles of helpful consultant and down-to-earth robot arm. This team-based interface concept was chosen to maximize user performance and comfort in controlling the inherently complex mechatronic technology. This paper describes our design decisions and rationale.

\section{Introduction}

Assistive Rehabilitation Robotics, a niche within Service Robotics, focuses on applications that allow a person with a severe physical disability to perform Activities of Daily Living (ADLs) and vocational support tasks that would otherwise require a human attendant. The robot is under control of its operator, for example by voice and head motion inputs to a personal computer running a Graphical User Interface (GUI) task design/execution program. Typical tasks for an assistive robot are handling books, medication, paper, computer media, food and drink, as well as controlling communication devices such as a phone or fax machine and activating electrical appliances through a computer-controlled Environmental Control Unit (ECU). The ProVAR development project's goal is to implement a system architecture that facilitates task construction and execution by nontechnical operators and integrates sensor feedback to environment interaction and object handling. These two separate functions are handled by two ethernetlinked computers: the one contains the user interface, and the other the real-time operating system for motion control.

\section{User Demographics}

The primary users for a robot such as ProVAR are individuals who have a physical disability but can communicate clearly and have normal cognitive ability, such as with high-level spinal cord injury (SCI) and cerebral palsy. The ProVAR Project is focused on users with quadriplegia from high-level (C1-C6) spinal cord injury.

There are about 89,000 individuals with quadriplegia as a result of a spinal cord injury in the United States today [1], with an estimated 3,000 new injuries resulting in quadriplegia each year. Most injuries (61\%) occur between the ages of 16-30. After injury, only $12 \%$ of this population return to vocational activity. Life expectancy is close to normal, which translates to a long-term need for technology aids and attendant care. Overall, it is estimated [2] that there are 150,000 people in the U.S. today who have severely impaired upper extremity function from all disabilities and who could be candidates for using a robotic assistant. Attendant care costs continue to rise as technology costs decrease. The ability to amortize inherently expensive independence and quality-of-life solutions such as modified vans, electric wheelchairs and assistive robots will continue to become easier in the future [3]

\section{Background}

The precursor to ProVAR was DeVAR, the desktop vocational assistant robot [4]. The two share a common geometry: a small robot arm mounted on an overhead track suspended above a desk worksurface. DeVAR had a simple GUI with a discrete-word, userspecific voice recognition system, and no provision for the user changing or adding tasks. All tasks were position-based, since the robot had no force sensing. The tasks, as a result, were "brittle", not robust, since any small difference in position of an object would cause the task to fail [5]. ProVAR is designed to circumvent such problems through inherently force-based object-manipulation. DeVAR's interface used only voice as a means to provide commands and receive feedback. ProVAR's interface will allow any commercially available assistive technology to be used for the physical interface to operators, assuring a customized, optimized fit to each operator's individual needs.

Several other rehabilitation robots exist, notably MASTER/RAID [6]. It is similar in concept and capability to DeVAR, but is set up in its own separate workspace adjoining the user's workstation. The entire system, therefore, requires twice as much floor space as DeVAR. Extensive user trials have been performed, which are leading to continued develop- 
ment. For example, subsequent research added an experimental graphical user interface to RAID [7]. Other assistive robots are in development or production, but have different geometries. The commercially available MANUS wheelchair-mounted robot allows motion-level control of manipulation in unstructured settings [8]; the Movaid R\&D project is developing an autonomous mobile robot for home use [9]. For recent reviews of rehabilitation robotics, see [10], [11].

\section{ProVAR Geometry and Design System components}

The ProVAR system consists of a Stauibli (Duncan, SC) PUMA-260 manipulator mounted on an overhead, $1 \mathrm{~m}$ long transverse track (see figure 1). This arrangement allows the robot to access objects and appliances on the desktop and on shelving on both sides, and to bring objects, such as a mouthstick, cups or telephone receiver near the user's face. The arm carries an Otto Bock (Minneapolis, MN) Greifer ${ }^{\mathrm{TM}}$, a parallel-jaw device with pinch, grasp and hook prehension. The Greifer, originally designed as a human hand prosthesis, has been augmented with touch sensors and an optical encoder for use as a robot gripper.

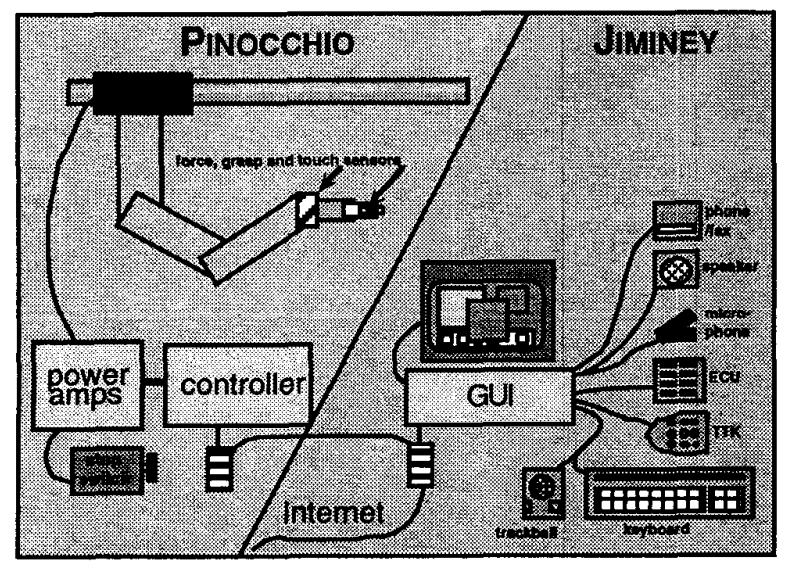

Figure 1. ProVAR System Components

The Greifer is outfitted with force-sensitive resistors (FSRs) embedded in the three pads of one finger. These FSRs (Interlink Electronics, Camarillo, CA), have an inverse log-log relationship between normal force and resistance. Interface circuitry gives an approximately linear relationship between force and voltage. In addition, an optical emitter/detector pair is mounted on the two fingertips of the gripper to detect when an object is between the fingers (this is essential for manipulating objects such as sheets of paper). The forearm of the robot is instrumented with four capacitive proximity sensors that have a sensing range of 100 to $150 \mathrm{~mm}$ [12]. These proximity sensors improve user safety and allow more robust manipulation by detecting unknown obstacles.
Since the functions of ProVAR extend to communication and environmental control, the workstation interface computer has connectivity solutions for the office: phone, fax, and Internet links are available under the Windows-NT operating system. A handsfree speakerphone allows delay-free phone use, while a robot-manipulated handset allows private conversation. An X-10-based ECU (Tandy Corp., Fort Worth, TX) allows users to operate nearby lights and appliances that have been outfitted with X-10 control boxes.

\section{Safety considerations}

Unlike a factory environment in which robots are confined to person-free zones, an office workstation implies that the operator and other individuals may share space with the manipulator. Safety considerations therefore dominate the design of the robot. The workspace of a PUMA-260 is small $(-50 \mathrm{~cm}$ radius quasi-spherical workspace). While this is too small to be useful in an office, a larger, stronger manipulator would have reduced the safety margins substantially. Augmenting the small arm with a linear track provided an appropriate solution for this environment. The PUMA was chosen for its anthropomorphic design and absence of external wiring. The Greifer end-effector was chosen for safety reasons: the hand prosthesis was designed to be used by humans in home and office environments for exactly the types of tasks ProVAR was designed to perform. Available robot grippers designed for industrial applications are not appropriate in our context of use.

\section{Interface Design Interface Layers}

The interface has several design layers: the physical layer provides the user with access to the operating system of the interface computer. For people with a physical disability, the solution is unique to each person's function and preference. The team of occupational therapist and rehabilitation engineer provides individualized worksite accommodations. For the ProVAR target user population, typical solutions include a voice recognition system for navigation of the operating system and robot control software, and a head motion tracking system for cursor control. Currently, NaturallySpeaking TM (Dragon Systems, Newton, MA) and the HeadMaster ${ }^{\mathrm{TM}}$ (Prentke-Romich, Wooster, $\mathrm{OH}$ ) provide these two functions on ProVAR.

The second interface layer is the operating system software. ProVAR's computer uses Windows-NT on a $266 \mathrm{MHz}$ Pentium-II system. Windows-NT allows access to all communication and manipulation functions through standard software tools and protocols.

The third layer of robot interface is the ProVAR simulation/preview environment written in HTML, 
Java and VRML, and accessed through a WWW browser (see figure 2). This layer communicates through a dedicated ethernet link to the control computer to send robot commands and receive status information. The operator navigates through hierarchical menus (written in Java) to select options and build a task. Icons represent the individual phases of each task, summarized on a storyboard [13]; popup menus from the icons permit default motion parameters to be edited (speed, via-points, sensor system set-up, etc.). For location specification, the on-screen depiction of the robot (a VRML model) can be manipulated with the cursor, as can movable objects in the workstation. A task can be previewed before being sent to the robot controller.

\section{Remote operation}

The robot is intended to be operated from the workstation, and motion is supervised by the primary user for reasons of safety. However, there are several scenarios that mandate remote operation. First, a user may already have a laptop computer personally configured and wheelchair-mounted. Since ProVAR's interface uses WWW-standards and is therefore designed to be platform independent, the main interface computer can communicate to any person's laptop computer either through an infrared link or through a secure Internet conection. The user can benefit from ProVAR's large monitor while control is mediated by the laptop computer.

A second scenario involves telediagnostics. ProVAR has a "Virtually-in-Person" WWW-controllable video capability termed VIP [14]. Using a remote browser, authorized technical support personnel can view the status of ProVAR, activate its functions remotely, and query the status of its sensors. This intervention is coordinated with the local user so that a solution to the problem being diagnosed can be made collaboratively and safely.

\section{Dual character user-interface concept}

The theory of Social Responses to Communication Technologies (SRCT) explains that an individual's "interactions with computers, television and new media are fundamentally social and natural, just like interactions in real life" [15]. SRCT is explicit people's interactions with technologies such as computers are not just similar to those between humans, but, on a fundamental level, are identical to them. Social rules that a person follows and expects others to follow in human-human interactions are directly applicable to human-computer interactions.

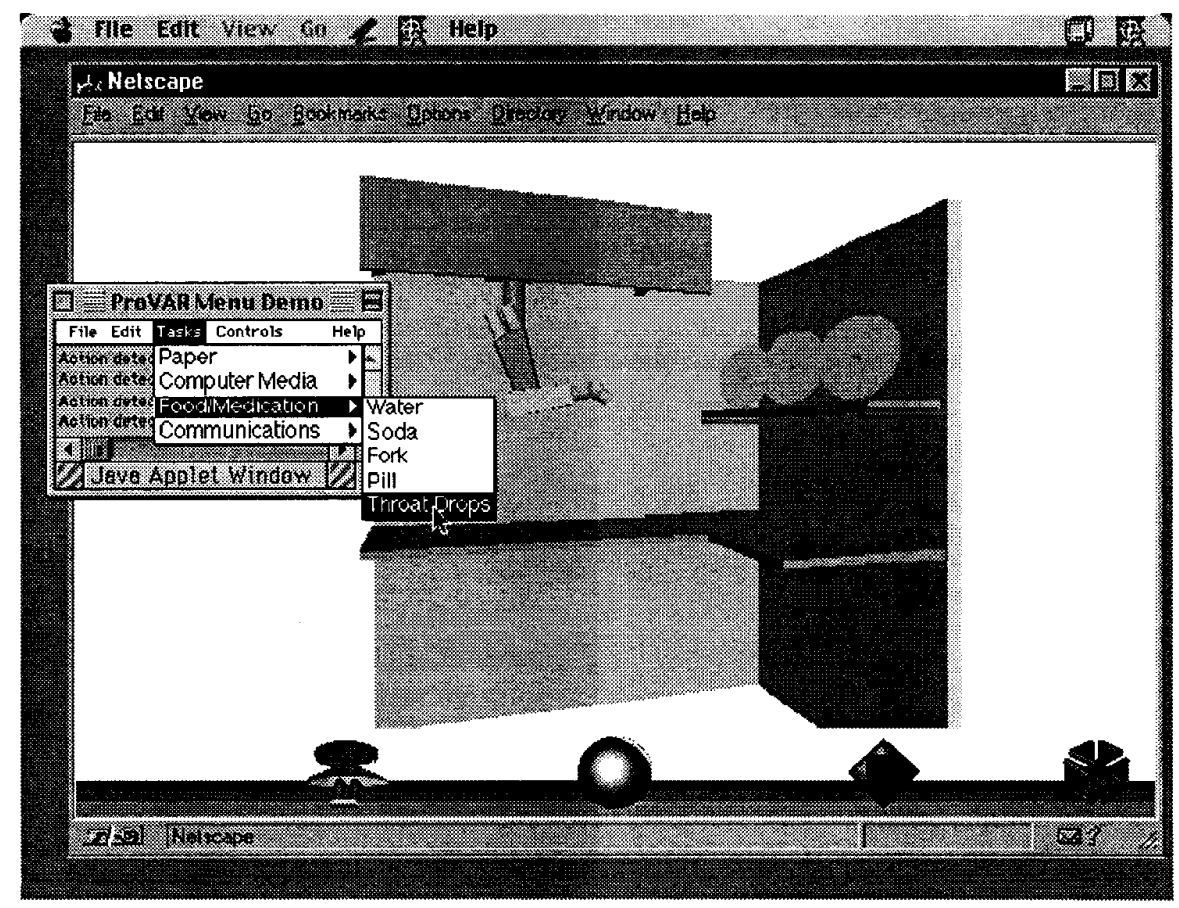

Figure 2. VRML Model of Robot Workstation 
A second design feature exploited in the ProVAR interface concept is the team-approach to design, learning and problem-solving [16]. Studies in engineering and social sciences have shown the benefits of teams: being on a team engenders a number of beneficial effects on the attitudes, behaviors and performance of individuals. People cooperate more with teammates and conform their behavior to match that of the groups.

The insights provided by SRCT are directly transferable to robotic user interface design, since SRCT implies that people will be treating the ProVAR system as a social entity. ProVAR's robot interface concept [17] is centered on the premise that a social entity consists of both Soma and Psyche components.

$$
\text { Social entity }=\text { Soma }(\text { body })+\text { Psyche }(\text { mind })
$$

If something exhibits primarily only one trait then the other is implied and proactively filled in by the enduser or viewer. Communication technologies manifest the psyche through the use of language. Robotic technologies will manifest the soma through physical embodiments and/or active deliberate movements. Thus while the user will interact with the computer-user interface as a social entity, the robot arm itself could be viewed as a separate social entity in its own right.

The ProVAR system follows this natural conceptualization by being divided into two parts: characters named Pinocchio (the robotic arm) and Jiminey (the GUI). The division between Jiminey and Pinocchio [18] is "artificial" because in reality the ProVAR system incorporates a number of computers that all have a role in the control of the robot. Where to draw the line between the two characters depends primarily on the surface or cosmetic structure of the user interface and a framing of the system topology to the end-user.

The user, Pinocchio, and Jiminey will work together to complete the desired tasks (figure 3). By leveraging natural social behavior, the quality of the interaction between the user and the robot interface can be enhanced. Regardless of how robust the components of the system are, there are many failure modes and difficulties that may arise in a semi-structured workstation setting. In the past, difficulties of even a very simple technical nature, such as the gripper power cable becoming unplugged, provoked a call by the end-user for on-site technical support. An interface design that casts the computer as a coach is effective in a problem-solving scenario. In the case of the ProVAR system, a pattern of corrective, proactive problem solving can be developed between user and computer.

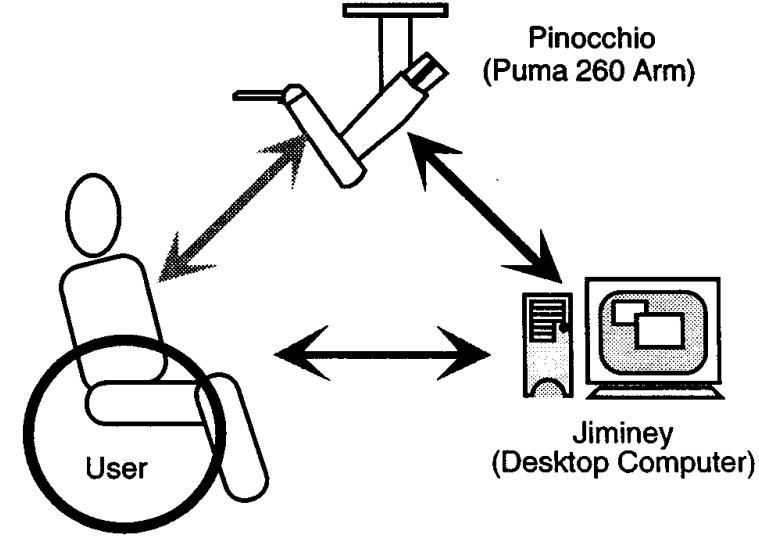

Figure 3. Lines of Communication among the teammates

\section{Controller Design \\ Design implementation}

The original PUMA-260 controller has been modified using Trident Robotics' hardware boards (Minneapolis, $\mathrm{MN}$ ) to allow real-time control from a personal computer, while using the original controller's amplifiers, safety circuitry, and arm cabling. In this design, the servo process in a PC communicates with the robot hardware via a TRC-006 interface board (ISA) and a TRC-004 open-architecture control board (figure 4). An 8-axis PC control board by Servo-To-Go (South Bend, IN) is scheduled to replace these two hardware boards to simplify the low-level communication and integrate the control of the track and gripper with that of the six joints of the arm. Also, an ATI Mini-40 F/T force sensor (Garner, NC) is mounted at the PUMA-260's wrist and communicates with the servo process via ATI's A/D board.

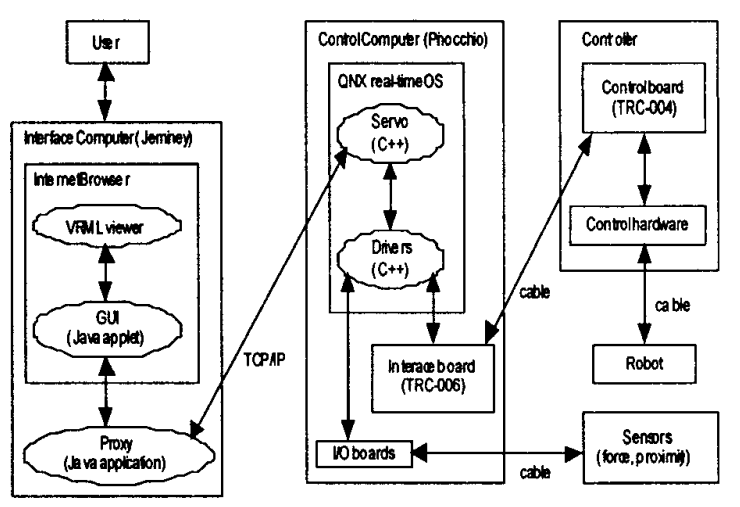

Figure 4. Overall Hardware/Software Architecture 
Communication between the controller PC and the proximity and touch sensors occurs over a two-stage network (figure 5). The first stage is an industrystandard CAN bus, which connects the CAN nodes to the PC through an ISA card with an on-board processor. This processor manages the data flow over the CAN network, thus relieving the $\mathrm{PC}$ processor of those duties. The information from the CAN nodes is received by the on-board processor and loaded into dual-port RAM, which can be accessed by the PC processor. The second stage of the network is the communication between the CAN nodes and the individual sensing elements. The Cheetah-1 CAN nodes (Zanthic, Alberta, Canada) communicate with the sensors through a synchronous serial (SPI) interface to distributed, addressable A/D converters. Both CAN and SPI buses can operate at $1 \mathrm{Mbit} / \mathrm{sec}$, and can therefore provide overall sample rates of at least $500 \mathrm{~Hz}$ for all sensors.

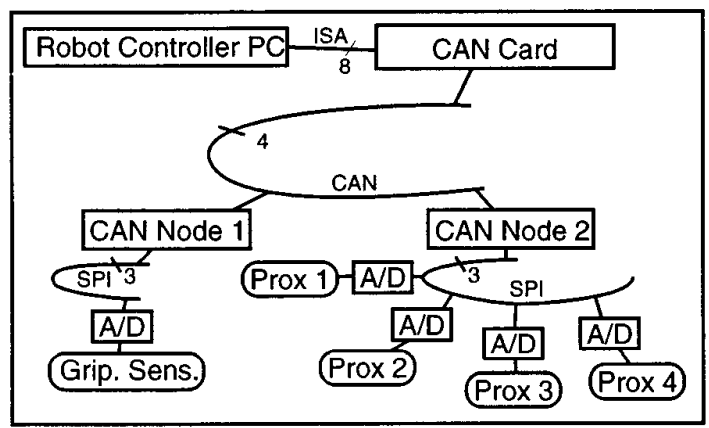

Figure 5. Sensor Network

\section{Real-time software implementation}

A general robot library has been developed to provide efficient and essential functions to simulate and control serial open-chain robotic mechanisms. This library is written in $\mathrm{C}++$ and compiled with the Watcom $\mathrm{C} / \mathrm{C}++$ compiler. Some of the implemented functions are matrix operations, forward kinematics, joint space forward and inverse dynamics, smooth motion trajectory (linear and cubic) generation, PID control, inter-process communication based on TCP/IP sockets, real-time timer routines, robot safety routines, and various drivers for low-level hardware (e.g., encoder, $\mathrm{D} / \mathrm{A}, \mathrm{A} / \mathrm{D}$, force sensor) communication.

Using this library, the main servo process simulates and controls the desired robot dynamic behavior during each sampling period while communicating with the interface computer and the robot hardware for new requests and updated sensor values. This on-line (real-time) simulation provides verification and confirmation of the intended control for higher-level safety checks. The servo process is executed continuously and synchronously in a $266 \mathrm{MHz}$
Pentium II PC running the QNX real-time operating system (Toronto, Canada) at a $500 \mathrm{~Hz}$ sampling rate, ensuring stable and safe robot behavior.

\section{Control structure implementation}

To provide intuitive control of redundant robotic systems and improve the ease of user interaction, ProVAR uses the control structure based on the operational space formulation [19]. In this framework, redundant robotic systems can be modeled and controlled at the object level. This allows users to specify desired object motions and forces in a more natural object coordinate system without explicitly dealing with the dynamic coupling effect of the actual robots. Users can adjust the stiffness of the controller in different directions of the object coordinate system. This is a powerful safety feature for interactive robots since, by reducing or eliminating control stiffness along some directions, the effects of unexpected impacts along the specified directions are diminished.

This control structure naturally integrates the use of artificial potential fields [20] for real-time collision avoidance for safety. Using a model of the environment and the proximity sensors, repulsive forces are generated from the objects in the environment. These additional forces are added to the control structure to repel the robot ensuring real-time collision-free motion while executing the desired task.

\section{Evaluation Concept}

\section{Discount assessment}

Nielsen's "discount usability testing" method [21] entails in-depth testing with a small number of subjects in a heuristic evaluation of system function, essentially turning test subjects into evaluators as they explore the interface under the guidance of the evaluator. This method was developed as an explicit answer to the practical impossibility and actual failure of laboratory-based testing techniques - adequate for simple interfaces - to predict the performance of complex commercial computer software products. Results from comparative methodological studies have shown that using Nielsen's testing method with only six users will yield approximately $80 \%$ of a complex system's usability problems, including over $95 \%$ of the most serious ones, as rated by system experts. Testing ProVAR with this method has the highest probability of useful results, given the timeframe of the project and availability of test subjects.

\section{Conclusion}

ProVAR's mechanical design provides a safe robotic assistant in a semi-structured office workspace setting for a person with a severe physical disability. The user interface has design provisions for handling inherently complex interactions through team-based problem-solving approach concepts. The real-time 
controller and sensor environment build on the experience of previous DeVAR generations to allow robust interactions with the environment in the face of calibration errors and unforeseen situations. Followon evaluation studies with this design will seek to validate the design choices and provide guidance toward the development of a commercially and technically viable rehabilitation robot product.

\section{Acknowledgments}

This project is funded by a Merit Review development grant from the U.S. Department of Veterans Affairs Rehabilitation Research Service, with additional support from the Stanford University departments of Mechanical Engineering and Computer Science.

\section{References}

1. S.L. Stover, P.R. Fine, Spinal cord injury: The facts and figures, The University of Alabama at Birmingham. 1986.

2. C.A. Stanger, M.F. Cawley, Demographics of rehabilitation robotics users. Technology and Disability, 5:2, 1996, 125-137.

3. H.F.M. Van der Loos, S.J. Michalowski, J. Hammel, L.J. Leifer, H.G. Stassen, Assessing the impact of robotic devices for the severely physically disabled. Proceedings First Inernational Workshop on Robotic Applications in Medical and Health Care, Ottawa, Canada, June 23-24, 1988, 23-25.

4. H.F.M. Van der Loos, VA/Stanford Rehabilitation robotics research and development program: Lessons learned in the application of robotics technology to the field of rehabilitation. IEEE Transactions on Rehabilitation Engineering, Vol. 3, No. 1, March, 1995, 46-55.

5. S.J. Sheredos, M.E. Cupo, Final Report, DeVAR Evaluation by the VA Technology Transfer Section, Baltimore, MD, Sept., 1994.

6. R. Cammoun, J.-M. Détriché, F. Lauture, B. Lesigne, Clinical evaluation of the MASTER robot system and development of a new version. Robotica, 11, 1993, 535-539.

7. J.L. Dallaway, R.M. Mahoney, R.D. Jackson, R.G. Gosine, An interactive robot control environment for rehabilitation applications. Robotica, Vol. 11, Part 6, Nov-Dec, 1993, 541-551.

8. H.H. Kwee, J.J. Duimel, J.J. Smits, A.A. Yuinhof de Moed, J.A. van Woerden, L.W. van de Kolk , J.C. Rosier, The MANUS wheelchairborne manipulator: system review and first results. Proc. 2nd Workshop on Medical and Health Care Robotics, Newcastle upon Tyne, UK, 1989.
9. E. Guglielmelli, Mobile robots in residential care. Proc. ROBOmed'94, 1994, 89-103.

10. H.F.M. Van der Loos, J.M. Hammel, R.F. Erlandson, eds., Special section on rehabilitation robotics. IEEE Transactions on Rehabilitation Engineering, 3:1, March, 1995.

11. R.M. Mahoney, ed., Special issue on robotic applications in rehabilitation. Technology and Disability, 5:2, 1996.

12. J.M. Vranish, Guiding robots with the help of Capaciflectors. NASA Tech Briefs, March, 1997, 44-48.

13. D.S. Lees, L.J. Leifer, A graphical programming language for robots operating in lightly structured environments. IEEE International Conference on Robotics and Automation, Atlanta, GA, May 2-7, 1993.

14. L.J. Leifer, S. Stepper, M. Schaefer, H.F.M. Van der Loos, VIPRR: A virtually-in-person rehabilitation robot. Proceedings 1997 International Conference on Rehabilitation Robotics, Bath, U.K., April 14-15, 1997, 79-82.

15. B. Reeves, C. Nass, The Media Equation. CSLI Publications, New York, 1996, p. 5.

16. D. Abrams, M. Wetherell, S. Cochrane, M.A. Hogg, J.C. Turner, Knowing what to think by knowing who you are: Self-categorization and the nature of norm formation, conformity and group polarization. British Journal of Social Psychology, June 1990, 29:2, 97-119.

17. J.J. Wagner, H.F.M. Van der Loos, L.J. Leifer, Dual-character based user interface design for an assistive robot, Proceedings ROMAN-98 Conference, Kagawa, Japan, 9/30 - 10/2 , 1998.

18. C. Collodi, Avventure di Pinocchio. Giornale per $i$ bambini, Year 1, n. 1, July 7, 1881 .

19. O. Khatib, A unified approach to motion and force control of robot manipulators: the Operational Space formulation. IEEE Journal on Robotics and Automation, 3:1, 1987.

20. O. Khatib, Real-time obstacle avoidance for manipulators and mobile robots. International Journal of Robotics Research, 5:1, Spring 1986, 90-98.

21. J. Nielsen, The art of navigating through hypertext. Communications of the ACM 33(3), March, 1990, 296-310. 